\title{
Styles de direction et fonctionnement des lycées
}

Robert Ballion

\section{OpenEdition}

\section{Journals}

Édition électronique

URL : http://journals.openedition.org/ries/4211

DOI : 10.4000/ries.4211

ISSN : 2261-4265

\section{Éditeur}

Centre international d'études pédagogiques

\section{Édition imprimée}

Date de publication : 1 décembre 1994

Pagination : 125-138

ISSN : 1254-4590

\section{Référence électronique}

Robert Ballion, "Styles de direction et fonctionnement des lycées », Revue internationale d'éducation de Sèvres [En ligne], 04 | 1994, mis en ligne le 17 avril 2015, consulté le 06 janvier 2020. URL : http:// journals.openedition.org/ries/4211 ; DOI : 10.4000/ries.4211

Ce document a été généré automatiquement le 6 janvier 2020

() Tous droits réservés 


\title{
Styles de direction et fonctionnement des lycées
}

\author{
Robert Ballion
}

1 L'analyse comparative du fonctionnement de vingt-cinq lycées constituant un ensemble où les diverses catégories administratives, géographiques, sociales de ce type d'établissement sont représentées, nous amène ${ }^{1}$ à confirmer ce que les travaux sur l'«école efficace $»^{2}$ ont bien montré, déjà depuis plus d'une décennie dans les pays anglo-saxons ${ }^{3}$ et plus récemment dans le nôtre. Toutes choses égales par ailleurs, c'està-dire en comparant des établissements similaires quant à leurs caractéristiques contextuelles, de composition, de structures qui constituent un faisceau de détermination de même nature, l'on observe que ces établissements se distinguent par la qualité de leurs résultats : certains établissements sont efficaces, ont des résultats supérieurs à ceux que l'on pouvait prévoir, d'autres ont une activité qui débouche sur des résultats conformes à la prévision, d'autres enfin peuvent être considérés comme contre-productifs dans la mesure où ils font moins bien que ce qui était attendu, compte tenu de l'espérance de réussite de leurs élèves.

2 Les synthèses de ces travaux permettent de dégager un certain nombre de facteurs que l'on rencontre généralement dans les établissements performants ${ }^{4}$. Dans cette constellation de facteurs d'efficacité, l'un joue un rôle prépondérant par son action fédérative, catalysant, c'est le style de direction de l'établissement.

Dans les pays où le chef d'établissement peut, par les compétences attachées à son statut, exercer un véritable leadership éducatif, l'influence du rôle de la direction sur le rendement de l'école a été d'emblée mise en évidence ${ }^{5}$. Il en a été autrement en France, où non seulement le chef d'établissement n'exerce pas une autorité pédagogique sur les producteurs directs du service éducatif que sont les enseignants, mais où il est soumis à une tension qui peut être paralysante quand elle est vécue comme une injonction paradoxale ${ }^{6}$. Puisqu'il est sommé en tant que représentant de l'État dans l'établissement (chef d'un service déconcentré) de se soumettre aux directives de sa hiérarchie et, qu'en même temps, par sa fonction d'organe exécutif de ce même établissement (chef d'un établissement public local) on l'invite à assumer ses 
responsabilités et son autonomie. Dans notre système éducatif, l'importance du rôle du chef d'établissement est moins nettement soulignée, mais comme ils « ont tendance à s'emparer de toutes les possibilités d'agir et à s'engager personnellement et pleinement dans la vie de leur établissement $\aleph^{7}$, l'impact de leur action sur la qualité du service produit par l'organisation qu'ils dirigent est noté dans toutes les recherches menées sur le fonctionnement des établissements.

4 F. Dubet et alii ${ }^{8}$, dans leur étude de trois collèges en rénovation, constatent que la mobilisation des enseignants qui leur apparaît comme le facteur prépondérant d'efficacité, est très largement dépendante de l'action personnelle du chef d'établissement. R. Ballion et alii ${ }^{9}$, constatent que les caractéristiques du style de direction tiennent une place importante dans l'explication des réussites différenciées des lycées qu'ils étudient, non seulement parce que leurs proviseurs ont une efficience inégale, mais aussi parce que la conception qu'ils se font de ce que doit être leur rôle est plus ou moins adaptée à la situation présente de l'établissement qu'ils dirigent. En particulier, un style de direction qui, hors contexte, est présenté comme un modèle : celui du proviseur démocratique, respectueux du libre arbitre de ses «collègues » et faisant confiance à la persuasion et à l'exhortation pour influer sur la conduite de ces derniers, apparait comme "contre-productif ", renforçant les dysfonctionnements de l'établissement, dans les lycées à la recherche d'un accord collectif.

O. Cousin et J.-P. Guillemet ${ }^{10}$, qui font porter leurs analyses sur deux groupes de lycées : un dont les performances (mesurées par les indicateurs de résultats au baccalauréat construits par la DEP) sont en hausse, et l'autre qui, au contraire, réunit des établissements " en baisse ", constatent que dans les établissements qui progressent, les équipes de direction sortent d'une action définie autour des tâches administratives pour s'impliquer plus ouvertement dans l'action pédagogique.

6 J.-P. Obin ${ }^{11}$ montre la nécessité qu'un établissement soit piloté et ne voit que le chef d'établissement qui soit en mesure d'assurer cette fonction, il est, dit-il, « le seul pilote possible du projet ». A. Henriot-Van Zanten et J. Migeot-Alvarado ${ }^{12}$ font le constat, en examinant l'implication des parents dans le fonctionnement de l'établissement, que le facteur prédominant de la participation des familles est l'impulsion donnée par le chef d'établissement.

\section{Une inégalité de réussite}

7 Tous les lycées sont confrontés, depuis la fin des années quatre-vingt qui ont vu en cinq ans, de 1985 à 1990, l'effectif du second cycle général et technologique croitre de plus de $30 \%{ }^{13}$, aux même défis : celui de s'ajuster à l'hétérogénéité scolaire accrue de la population d'élèves accueillie et celui de prendre acte, pour en contrebalancer les effets négatifs, du nouveau type de relations que les jeunes tendent à établir, d'une manière plus généralisée qu'antérieurement, avec l'institution éducative : désengagement, repli individualiste, attitude passive et critique de " consommateur ».

8 Le poids de ces contraintes est fortement différencié selon les catégories sociales d'établissement. Dans notre échantillon de vingt-cinq lycées, certains d'entre eux, situés dans des zones périurbaines déshéritées, dans ces banlieues que l'on peut appeler " quartiers d'exil $»^{14}$, subissent de plein fouet le changement. Plus de la moitié de leurs élèves sont ces "nouveaux lycéens $\|^{15}$ qui, avant la fortune sociale du mot d'ordre « $80 \%$ d'une génération au niveau du baccalauréat», n'auraient pas accédé en 
seconde ${ }^{16}$. Peu armés par leurs antécédents scolaires, par le fait qu'ils n'ont aucune idée de ce qu'implique le suivi d'études secondaires, à affronter les exigences de l'institution à ce niveau, après une brève période d'euphorie à leur entrée en seconde, ils « décrochent » très vite et se maintiennent dans un rapport magique à leur avenir. Ce sont de nouveaux élèves, qui ne savent et ne veulent travailler, et n'en voient pas la nécessité, car leur expérience du collège les a convaincus de l'automaticité du déroulement d'une scolarité conçue comme l'écoulement d'un flux, et les "analyses coûts-avantages" qu'ils effectuent d'une manière spontanée les incitent rationnellement à ne pas trop investir pour accéder à un bien (le baccalauréat) qui, en se généralisant, a perdu beaucoup de sa valeur ; élèves pour lesquels il faut, si l'on ne baisse pas les bras, concevoir et mettre en œuvre une pédagogie de prise en charge bien éloignée des pratiques professionnelles des enseignants de lycée. Ce changement de population va de pair avec un changement de climat. Car les élèves de ces établissements « sensibles", où des enseignants nous disent « autrefois, on ne pouvait pas enseigner, maintenant on ne peut plus vivre... », ne se comportent pas en lycéens, ne se soumettent pas au code élémentaire régissant les rapport humains dans l'enceinte scolaire. La cité pénètre de plain-pied dans l'établissement, par les élèves qui se plient au modèle identitaire que sécrètent leurs conditions de vie et par les "éléments extérieurs " qui considèrent le lycée comme un équipement ouvert, où s'organisent toutes les activités licites ou illicites de la jeunesse. La violence, les conduites délictueuses qui, nous dit-on, « feraient qu'à l'extérieur on appellerait PoliceSecours, mais que dans l'établissement on est obligé de subir ", plus largement la disparition de toute crainte de sanctions sociales, font de ces lycées au bord de l'explosion, des établissements qui ont beaucoup de mal à garder leur statut de structure de formation ${ }^{17}$.

9 Lorsque l'on passe des lycées périurbains des grandes agglomérations aux lycées de «ville», des établissements où les enfants des milieux populaires sont largement majoritaires à ceux où la population d'élèves se rattache massivement aux couches moyennes et supérieures, le degré de contrainte baisse, on ne parle plus de crise, de risque d'explosion, mais de processus délétère d'implosion, de perte d'identité.

10 Dans ces établissements, qui pour beaucoup d'entre eux se sont vu adjoindre, par la modification de la carte scolaire tenant à l'ouverture de nouveaux lycées, le recrutement de collèges situés hors de leur zone traditionnelle de desserte du centreville, l'on estime à 20 à $30 \%$ le taux d'élèves dont le profil scolaire ne correspond pas à celui qui est attendu - par les producteurs du service éducatif - pour tirer profit de l'enseignement secondaire long. Ces élèves, non seulement, ne sont pas majoritaires, mais, contrairement à leurs condisciples des banlieues "chaudes", sont, eux, "gentils", ne tentent pas de subvertir violemment le fonctionnement de l'institution. Mais si leur comportement n'est pas d'opposition, il est de fuite, et si leur poids numérique est relativement faible, l'impact de leur conduite l'est beaucoup moins, non seulement parce qu'il suffit d'une minorité qui ne joue plus le jeu, pour que le « ça va de soi » sur lequel repose le contrat éducatif perde de sa pertinence, mais aussi parce que ce type de conduite devient un modèle auquel se conforment d'autres élèves qui, pourtant, auraient capacité à jouer convenablement le rôle de lycéen.

11 Confrontés à l'absentéisme massif de certaines catégories d'élèves, à celui sélectif mais aussi perturbant ${ }^{18}$ de beaucoup d'entre eux, à la baisse du travail personnel, aux manifestations de plus en plus fréquentes de «mal vivre» de la jeunesse par des 
conduites de fuite et de dépendance que traduisent le tabagisme, la consommation d'alcool, de drogue, par aussi le développement de troubles psychologiques, ces lycées sont placés devant la nécessité de concevoir des modalités de prise en charge globale du jeune, seules capables de remédier à la perte du sens de la condition de l'élève.

Ce sens reste encore intact et peut-être même s'est confirmé, car la scolarisation de masse a exacerbé la concurrence scolaire dans les lycées dits d' "excellence $»^{19}$ qui, selon la formule de F. Dubet (op. cit.), sont au « centre du centre». Ces établissements, relativement nombreux à Paris, mais présents aussi dans les banlieues ouest et sud de la capitale et dans les grandes villes de province, dont les résultats au baccalauréat se maintiennent autour de $90 \%$ d'admis, "jouent " comme nous le dit un proviseur «à guichet fermé », puisque le nombre de demandes d'entrées dont ils font l'objet dépasse largement leur capacité d'accueil. Ils reçoivent donc une population d'élèves scolairement triée, à bonne espérance de réussite, et semblent pouvoir se maintenir dans leur intangibilité d'appareil de production de l'élite. Mais, néanmoins, ils sont, même si ce l'est d'une façon atténuée, soumis eux aussi, au défi du changement.

La politique élitiste qui se traduit non seulement par le «filtrage » de l'entrée mais aussi par la " purification » à l'issue de la seconde ${ }^{20}$ en « orientant » les élèves dont les performances ultérieures risqueraient de faire baisser le taux d'admis au baccalauréat, est maintenant plus difficile à mettre en œuvre.

Ces établissements sont, malgré leur position en marge, soumis néanmoins à la pression symbolique des représentations dominantes portées par le discours public : obligation d'insertion faite aux établissements scolaires, évolution du rapport de force au bénéfice des usagers dans la procédure d'orientation. Qui plus est, l'ouverture de l'accès en seconde leur fait recevoir, eux aussi, davantage d'élèves qui vont se retrouver en situation d'échec relatif. Le nombre d'élèves potentiellement à « rejeter » devient alors trop important et risque de rendre visible et par là scandaleuse une pratique jusqu'alors tolérée.

15 Pour cette catégorie d'établissements, apparemment protégés mais en fait subissant eux aussi l'effet perturbant du changement, se pose de plus en plus, comme pour les autres lycées ${ }^{21}$, le problème de la réponse à apporter au désarroi des jeunes, qui, comme le remarque M. Gentzbittel (op. cit.) pour certains des élèves de son établissement bien représentatif de ces lycées "haut de gamme", prennent tout à coup conscience... "Qu'il n'est pas naturel de fréquenter le lycée $»^{22}$.

16 Dans chacun de ces groupements d'établissements, constitués en fonction de leur degré d'exposition aux changements que génèrent le développement du lycée de masse et la façon dont les jeunes ont tendance, maintenant, à vivre leur préparation à l'« entrée dans la vie", les lycées se partagent en deux catégories. Ceux qui subissent les dysfonctions sans avoir la capacité de mettre en œuvre des stratégies de remédiation, ou tout au moins, voient leur laborieuse et encore incertaine politique d'adaptation gagnée de vitesse par un processus de dégradation. Et ceux qui, au contraire, ont comme nous le dit un proviseur, "fait une véritable révolution culturelle », pris acte, sans gaieté de cœur, de la disparition d'un certain ordre institutionnel, culturel, et, non seulement opèrent les ajustements pédagogiques et éducatifs nécessaires, mais par la mobilisation qui permet cette mutation et qui est confirmée, nourrie par elle, sont entraînés dans une dynamique de progrès. 


\section{Le profil d'efficacité}

17 Les établissements que l'analyse nous a permis de qualifier d'établissements efficaces, qui ont une qualité de résultats, aussi bien académiques que relevant du climat de vie et de travail de la collectivité qu'ils constituent, supérieure à celle des établissements qui leur sont semblables du point de vue des ressources (financières, humaines, structurelles) dont ils disposent, ont trois caractéristiques générales de fonctionnement : un projet clair, ambitieux et programmatisé, une forte cohésion et mobilisation des personnels, et une prise en charge ouverte et incitative des élèves.

18 Cette manière de fonctionner est le fruit de l'action d'une variable générale, d'une méta-variable qui subsume les autres, qu'est la structuration. Ces établissements tendent à être des organisations intégrées, en surmontant la segmentation inhérente à la production du service éducatif dans notre pays, qui fait qu'un établissement scolaire est une collection de professionnels et d'usagers et non pas un collectif.

19 La circulation de l'information, la constitution de réseaux, la concertation qui génèrent de la cohésion, de la mobilisation et de la synergie, dépendent fortement de l'existence de données facilitatrices ou contraignantes qui relèvent de deux ordres de phénomènes : les structures et les mentalités.

20 L'importance des effectifs d'élèves et corrélativement d'enseignants, la structure pédagogique, la structure architecturale définissent des conditions plus ou moins favorables à la mise en œuvre d'une action à caractère collectif. Si un effectif jugé raisonnable d'élèves (ne dépassant pas mille deux cents), si l'homogénéité des structures pédagogiques (le lycée polyvalent reste encore à construire) ont un effet facilitateur notable, c'est la structure architecturale de l'établissement qui est toujours mise en avant pour rendre compte de la facilité ou de la difficulté à établir des relations, du face-à-face. Les lycées où la communication existe, où l'on dit que l'information circule très vite, où il est difficile pour les individus ou les groupes de se maintenir dans une position d'isolement, ont une structure architecturale concentrée, ramassée autour d'un espace central de dégagement, de passage et de rencontre. À l'inverse, les établissements qui n'arrivent pas à ce que les gens travaillent ensemble, sont handicapés par un éclatement, une segmentation de leur structure architecturale ou une disposition tout en longueur des bâtiments qui rendent très malaisées toutes possibilités de regroupements.

21 Les facteurs facilitateurs relevant des mentalités des acteurs ne renvoient pas à des dispositions personnelles qui concernent les conceptions de l'action éducative qu'ont les producteurs du service, de leurs orientations idéologiques, ou de leurs identités professionnelles par leur rattachement à un corps, à une discipline, mais à des données collectives qui exercent une pression sur les représentations et les conduites individuelles. Les établissements engagés dans une dynamique de progrès se réfèrent, et ceci d'une manière explicite qui fait que cette situation est clairement perçue par les acteurs, à une histoire et à une culture.

22 Les personnels de ces lycées exercent leur activité professionnelle dans un cadre, une organisation qui a sa "personnalité ", un "caractère propre » qui s'est maintenu en continuité depuis ses origines et qui engendre une espèce d'obligation statutaire: rester digne de ceux qui vous ont précédé; pour certains établissements, conserver l'esprit des "pionniers" qui ont créé le lycée il y a dix ou quinze ans; dans tous, 
maintenir la bonne réputation, la bonne image externe à laquelle aussi bien les adultes que les jeunes sont très attachés.

La cohésion, la mobilisation sont nourries par une culture d'établissement qui articule la recherche permanente d'innovation et le travail en équipe. Cette culture, qu'une formule reprise dans plusieurs de ces établissements résume : "Ici, l'on travaille... », a une capacité d'imposition très forte qui fait que tous les nouveaux venus y adhèrent ${ }^{23}$ et, fait important qui nous paraît devoir être souligné, n'est pas une culture idéologique (on ne s'interroge pas sur le fait de savoir si l'enseignement secondaire long n'est accessible qu'à une élite ou doit s'adapter à la masse). Les contraintes de la prise en charge de la nouvelle population et, plus largement, de l'évolution des attitudes des élèves, sont posées en termes de défis techniques, auxquels on répond positivement en s'appuyant sur une visée d'excellence professionnelle.

\section{La variable génératrice, le rôle de la direction, la nécessité d'un « patron ${ }^{24}$}

24 Les facteurs d'efficacité que nous venons d'évoquer ne produisent leurs effets qu'accompagnés par un certain style de direction que les Anglo-saxons qualifient de leadership éducatif. Sans l'existence d'une direction de cette nature, des données contextuelles comme l'effectif restreint et une structure architecturale propice aux interactions ne génèrent pas d'effets positifs, et des caractéristiques contingentes, comme une histoire et une culture elles-mêmes positives, peuvent très vite disparaître si la direction ne manifeste pas une vigilance, un pilotage adaptés qui permettent à l'établissement, malgré les bourrasques, de maintenir le cap, de "garder son âme " comme nous le dit un de ces proviseurs. À l'inverse, des établissements qui ne bénéficient pas de certaines des caractéristiques à effet positif peuvent, malgré tout, et ceci grâce à l'action de l'équipe de direction, réussir dans la voie de l'optimisation.

25 À la distinction que l'analyse des vingt-cinq lycées nous a permis de faire entre ceux qui subissent une situation et ceux qui, au contraire, en maîtrisent les effets négatifs et sont engagés dans un processus de changement positif, correspond la distinction entre deux styles de direction. Dans les lycées de la première catégorie, les proviseurs se réfèrent au modèle éprouvé de "l'accompagnateur ", disent qu'ils essaient de gérer en laissant les gens travailler, tout en restant le plus disponible possible. Ils se considèrent avant tout comme des "pourvoyeurs de ressources». Par contre les proviseurs des lycées qui "s'en sortent" ou tentent de "s'en sortir", ont un comportement ouvertement volontariste, revendiquent un style de direction qui fait d'eux, au-delà de la fonction de proposition, de soutien, d'organisation, l'acteur dont l'action structure et oriente l'activité de l'établissement.

Ce rôle de direction très actif ${ }^{25}$, assez souvent jugé par certains enseignants comme trop interventionniste ${ }^{26}$, que mènent ces chefs d'établissement, la plupart du temps bien en accord avec leur adjoint, est explicable par le fait trivial que les lycées ne peuvent plus être maintenant simplement administrés, ils doivent être dirigés.

Aucun des établissements dans lesquels nous avons enquêté, même si certains d'entre eux sont dans une situation très confortable par rapport aux autres, ne peut, comme c'était le cas pour le lycée traditionnel, «tourner tout seul ». Même les établissements de "haut de gamme", sont, non seulement confrontés à des dysfonctionnements 
inconnus il y a quelques années, mais ont à gérer des demandes qui leur interdisent de s'endormir sur leurs lauriers. Il en résulte que tous les lycées sont amenés à définir une politique. Or, pour que l'établissement mette en œuvre une politique, il lui faut accéder au rôle d'acteur collectif, ce qu'il peut difficilement faire étant donné la faiblesse de sa structuration interne et de son organisation. C'est donc le chef d'établissement qui par son statut est le seul à pouvoir incarner l'établissement dans sa globalité, qui est placé dans la nécessité d'agir, d'exprimer, au travers de son action, celle de la collectivité.

Les chefs d'établissement se doivent d'agir, car à l'exception de quelques établissements à faible effectif et à forte cohésion enseignante, il n'y a pas dans les lycées un accord général, ou tout au moins une position hégémonique, qui permette de dégager une ligne commune. Comme nous le dit J.-L. Derouet (1992, op. cit.), « l'école est irrémédiablement entrée dans un univers à justification multiple ». Il est par exemple frappant de constater que des grands lycées polyvalents qui fonctionnaient jusqu'à ces dernières années selon l'orientation pédagogique et idéologique de la promotion sociale par l'école, sont maintenant "désaccordés " et les producteurs du service (enseignants, CPE) attendent de la direction qu'elle tranche entre les options antagonistes qui se développent.

Les chefs d'établissement peuvent agir. Tout d'abord parce que tout un ensemble de changements institutionnels, quant à leur rôle et à leurs compétences (la dernière mesure à haute portée symbolique a été celle concernant la réglementation de l'orientation), a considérablement accru le poids de leur parole et leur a donné une autorité (capacité à imposer la contrainte) accrue. Ils peuvent aussi agir, car ils trouvent toujours dans leur établissement un groupe sur lequel s'appuyer, personnes "dynamiques ", "relais » qui se constituent comme un noyau ou un réseau et qui développent, collégialement, l'action impulsée par la direction.

Mais, comme le rappelle G. $\mathrm{Mamou}^{27}$ en citant le texte d'une circulaire d'un ancien ministre de l'éducation nationale : [...] «l'art de commander ne se réduit pas à la possibilité de donner des ordres mais à la capacité de les faire exécuter [...]. L'autoritarisme, c'est l'autorité sans discernement et sans dialogue ».

31 L'efficience de l'action du chef d'établissement, dans un de ses modes d'actualisation principal qu'est sa capacité à influer sur les producteurs directs de l'acte éducatif que sont les enseignants et les CPE, dépend de la légitimité qui lui est accordée et dépend aussi, ne craignons pas de le dire en gommant toute connotation péjorative, de son habileté manœuvrière.

Cette habileté ressort du «discernement » qu'évoque le ministre: savoir ce que l'on peut faire, ou ne pas faire; savoir utiliser d'une manière différenciée les possibilités offertes (action individuelle, collective, personnelle, institutionnelle ; "capital social » et compétences spécifiques des individus-relais) et de l'importance et la qualité du contact.

Quant à la légitimité, elle découle de deux ordres de considérations. L'un relève de l'impersonnalité inhérente à l'organisation : le chef d'établissement tire de plus en plus sa légitimité de ses compétences professionnelles et non pas de sa position statutaire dans une hiérarchie. L'autre, au contraire, est celui du registre de la relation personnelle, il ne s'agit plus du descriptif du poste mais de celui de la personne.

Ces proviseurs à l'autorité acceptée, sont tous considérés par leurs personnels comme des individus de grande qualité humaine : hommes et femmes de devoir, de conviction, 
de courage dans la prise de responsabilité et illustrant par leurs comportements une relation très humaniste aux autres.

Lorsqu'un enseignant nous dit, parlant de son proviseur : « il vendrait son âme pour un bon rapport...", nous ne serons pas par la suite étonné de voir que ce chef d'établissement, malgré ses qualités «techniques", malgré son engagement professionnel, n'arrive pas à impulser une dynamique collective, ne peut jouer son rôle de « locomotive » pour reprendre l'image de l'Inspection générale.

Analysant la façon dont ces chefs d'établissement conçoivent et exercent leur fonction, on dira que ce sont des politiques, des organisateurs, des techniciens et des éducateurs.

Des politiques. Ils le sont de la manière la plus haute en ayant une conception claire et formulée de la légitimité de leur fonction d'autorité. Ils apportent un grand soin à exposer les fondements sur lesquels s'appuie leur pouvoir. Ils sont les garants de l'expression de l'intérêt général et de sa mise en œuvre. Intérêt général qui se situe à deux niveaux d'émergence: le niveau étatique par le biais des règlements et des commandes institutionnelles, et le niveau local, celui de l'établissement en tant que communauté qui, par l'intermédiaire de son conseil d'administration, définit, dans la marge d'autonomie qui lui est concédée, sa propre «loi ». Ils ne se veulent pas des médiateurs qui arbitrent les rapports de force entre les différents " lobbies ", comme il nous l'a été dit, même s'ils interviennent de plus en plus, car ils sont sollicités par les élèves dans les conflits qui peuvent opposer ces derniers à des enseignants, mais se considèrent, selon une métaphore proposée par l'un d'entre eux, comme étant les "porteurs de la loi ", situés au sommet d'un triangle qui a à sa base: d'un côté les usagers, les élèves et leurs parents, et de l'autre les enseignants. Au-delà d'une aisance dans la gestion des relations humaines, d'une facilité et d'un goût pour le contact, ils maitrisent, plus ou moins consciemment, les techniques de production d'influence.

Des organisateurs. Nos chefs d'établissement «interventionnistes» ne sont pas uniquement des politiques mais aussi des organisateurs. Ils ne raisonnent pas en termes de mentalité, de bonne volonté, mais de structure et de dispositif. Il s'agit de mettre en place des « construits humains $»^{28}$ qui mettent en relation les acteurs dans un cadre et avec un objectif fixés, des procédures qui stabilisent et dépersonnalisent les actions et, plus largement, de traduire tout projet en programme d'actions, en mobilisation des moyens et en définissant et répartissant des rôles.

Des techniciens. Ces chefs d'établissement tirent leur légitimité non pas de leur position dans une hiérarchie, mais de leurs capacités techniques. Ils ont une conception très professionnelle de leur rôle : "avant, on se contentait de reproduire » nous dit un proviseur, «maintenant il faut innover ». Plus précisément, il pourrait ajouter, il faut posséder des compétences de gestionnaire, de manager et de pédagogue. Nous avons pu constater que, par exemple, concernant la rénovation des lycées, la plupart de ces chefs d'établissement ont joué, dans cette opération, un rôle d'expert pédagogique, expliquant ce que devait être un module, ce que devait être l'évaluation et comment organiser tout cela. Ces proviseurs ne se contentent pas de soutenir les enseignants de leur établissement qui s'engagent dans les innovations, ils proposent des idées, analysent les modalités de leur mise en pratique, se situent donc comme porteurs de compétences dans tous les domaines de la vie de l'établissement (c'est particulièrement vrai pour tout ce qui concerne la relation éducative aux jeunes), à l'exception des contenus des disciplines et de leurs didactiques. Cette compétence professionnelle multiforme contribue non seulement à donner du poids à leurs interventions, mais 
tend à faire passer les oppositions aux innovations du plan des valeurs (on est pour ou contre telle mesure en référence à la représentation que l'on a de ce que doit être l'enseignement) à une opposition tenant au degré d'acceptation d'une remise en cause des modalités de production du service éducatif. Cette opposition n'est plus présentée comme idéologique, mais comme instrumentale, et les opposants sont placés dans la situation inconfortable de professionnels qui refusent de remettre en question leur confort.

Des éducateurs. Ces chef d'établissement se comportent comme des chefs d'entreprise, mais à deux réserves près. L'organisation dont ils essaient d'optimaliser le fonctionnement a une mission d'intérêt général, et ils ne perdent jamais de vue que la finalité de son action est l'élève. Et des questions comme : à quoi cela sert de faire des sorties, des voyages, d'opérer des jumelages, de mettre en place un traitement informatisé des absences, de l'évaluation..., ils les formulent pour garder à l'esprit que la finalité du système n'est pas lui-même, mais l'élève.

Cette part accrue du chef d'établissement dans la détermination de la qualité du fonctionnement de l'organisation qu'il dirige, n'est pas sans poser de problèmes.

Celui tout d'abord de la congruence des situations. Correspondance entre le poids des exigences, des responsabilités qui pèsent sur ces acteurs et les gratifications en termes de traitement, de profil de carrière qui leur sont accordées, et, ressentie nécessaire peut-être d'une manière encore plus forte par les intéressés, correspondance entre l'invitation pressante qui leur est faite à faire donner pleinement leur capacité d'initiative et le degré réel d'autonomie concédée. «Qu'on nous fasse confiance ! » est une demande que nous avons souvent entendue, sans que ceux qui l'expriment rejettent de quelque manière que ce soit ce corollaire de l'autonomie qui est le contrôle de la conformité et l'évaluation des résultats.

Ces nouvelles compétences liées au rôle de chef d'établissement, il n'est pas sûr que, jusqu'à maintenant, elles aient été suffisamment prises en compte dans les critères de recrutement et dans les référentiels de formation, même si les responsables de l'administration qui ont en charge ces tâches ont conscience de la nécessité d'opérer ces ajustements.

L'évolution du rôle de direction contribue fortement, comme nous l'avons constaté, à accroître le malaise des enseignants, malaise qui non seulement traduit la difficulté qu'ont beaucoup d'entre eux à concevoir et mettre en œuvre les modifications de leur métier, qui est nourri par les changements intervenus dans leurs relations avec leurs élèves, mais qui aussi exprime l'incertitude et la crainte que suscite le chevauchement des compétences, par la mise en pratique d'une conduite de direction éducative. On peut estimer que cette tension n'est que transitoire, engendrée par le passage d'un état de fonctionnement qui est celui d'une "bureaucratie professionnelle " ${ }^{29}$, à un autre où l'établissement» ne peut plus être simplement administré mais dirigé et piloté. Il n'empêche que la gestion de cette transition soulève de nombreuses difficultés.

L'une des dernières que nous évoquerons et qui nous a frappés par son caractère de nouveauté, est l'accroissement des disparités entre établissements. Ces disparités ne tiennent plus seulement aux différences dans les ressources matérielles, humaines 
(caractéristiques socioculturelles des élèves, professionnelles des enseignants), mais aussi à la qualité de la direction. Il en résulte deux problèmes. Tout d'abord celui de réduire (par un recrutement plus ciblé, par la formation initiale, continue, par les retours de l'évaluation des chefs d'établissement) l'écart existant entre les deux catégories extrêmes de chefs d'établissement que sont, selon l'analyse de l'Inspection générale (op. cit.), d'une part, les proviseurs «locomotives» (35\%), de l'autre, ceux catégorie « heureusement peu nombreuse » $(10 \%)$ - qui « aggravent l'inertie ». Et faire en sorte que l'établissement soit en partie protégé des fluctuations liées à la mobilité (forte) des chefs d'établissement, en allant dans le sens d'une « dépersonnalisation » de la fonction et ceci en visant à généraliser certaines pratiques, en les inscrivant dans les profils de poste, et en s'efforçant à ce que l'organisation intériorise, stabilise dans son fonctionnement des processus qui reposent trop sur l'engagement et la personnalité de l'individu.

\section{NOTES}

1. La recherche a été réalisée pour le compte de la direction de l'évaluation et de la prospective (DEP) du ministère de l'éducation nationale, et est publiée sous le titre: Le lycée, une cité à construire, Paris, Hachette, 1993.

2. Pour un aperçu de cette orientation de recherche, voir : R. Ballion, La bonne école, évaluation et choix du collège et du lycée, Paris, Hatier, 1991.

3. Un des derniers bilans de cette orientation de recherche est proposé par J. Scheerens, Effective schooling, Londres, Cassel, 1992.

4. Voir Centre international d'études pédagogiques, La performance et l'efficacité des établissements d'enseignement secondaire, Sèvres, ministère de l'éducation nationale (France), ministère de l'éducation (Québec), 1990.

5. Pour un état des recherches dans ce domaine dans les pays anglo-saxons, voir: A. Grisay, Du mythe de la "bonne école " à la réalité (fuyante) de l'"école efficace», université de Liège, Belgique, 1988.

Voir aussi C. Hopes (sous la direct, de), Le chef d'établissement et l'amélioration du fonctionnement de l'école. Étude de cas de dix pays de l'OCDE, Paris, éd. Economica, 1988.

6. Ainsi, $71,5 \%$ des chefs d'établissements secondaires estiment qu'en ce qui concerne leurs relations avec le rectorat, les discours et les pratiques sont contradictoires au plan de l'autonomie des établissements. Source : B. Maresca ; O. Charrier, Le projet d'établissement, Paris, CREDOC, 1992.

7. M. Sellier, «L'Europe et ses chefs d'établissement ", Éducation et Management, n 10, nov. 1992.

8. Trois collèges en rénovation, Paris, Commissariat général du Plan, 1988.

9. «Fonctionnement des lycées : étude de cas ", Les Dossiers Éducation et Formations, n 10, DEP, ministère de l'éducation nationale, 1991.

10. "Variations des performances scolaires et effet d'établissement ", Éducation et Formations, DEP, ministère de l'éducation nationale, $\mathrm{n}^{\circ} 3$ 1, avril-juin 1992.

11. La crise de l'organisation scolaire, Paris, Hachette, 1993.

12. La participation des parents au fonctionnement des établissements secondaires, ministère de l'éducation nationale, DLC, 1992. 
13. 363000 élèves en plus, le taux de bacheliers qui était de 30,2\% en 1985 (253 000) est monté en 1990 à 44,4 \% (384 000), pour atteindre en 1992 51,5\% (434 000) et 54,7\% (457 810) en 1994.

14. F. Dubet, D. Lapeyronnie, Les quartiers d'exil, Paris, Seuil, 1992.

15. F. Dubet, Les lycéens, Paris, Seuil, 1991.

16. En 1985 , le taux d'une génération accédant en seconde était de $42,1 \%$; en 1990 , de $54,4 \%$. Le taux d'accès de troisième en seconde, qui était de 63,9 \% en 1985, s'est élevé à 70,7 \% en 1990.

17. Voir R. Ballion, Les lycées «sociaux": la «reprise en main», in: Violences, conflits et médiations, Migrants-Formation, CNDP, Paris, $\mathrm{n}^{\circ}$ 92, mars 1993.

18. Car, dit M. Gentzbittel (La cause des élèves, Paris, Seuil, 1991), avec l'absence, «toutes les fragilités de l'institution sont à l'occasion apparentes ".

19. Apprécions la profession de foi, par la bouche d'un ancien proviseur du plus célèbre d'entre eux : « Louis-le- Grand ! C'est le nom d'un roi illustre, mais aussi d'un lycée qui depuis quatre cent vingt-cinq ans s'est voué sans relâche à la recherche de l'excellence ", P. Deheuvels, L'excellence est à tout le monde, Paris, Robert Laffont, 1988.

20. Voir l'exemple de certains lycées parisiens dans R. Ballion, La bonne école, (op. cit.).

21. Un conseiller principal d'éducation compare l'expérience qu'il vit dans un lycée d' élite ", à celle qu'il a connue dans les lycées de banlieue. "Nos élèves qui sont issus à $95 \%$ de milieux favorisés, ont, d'un point de vue psychologique, les mêmes problèmes et les mêmes réactions que les lycéens des milieux défavorisés. À X, j'avais des cas sociaux; ici, les effets sont presque les mêmes quand on voit les familles essayer de régler leurs problèmes par l'argent. Ici, je vois des élèves qui viennent pleurer parce qu'ils se sentent en situation d'échec. »

22. Ce proviseur constate que pour ces jeunes que l'on pourrait croire bien engagés sur les rails de la réussite...« Chaque jour est une rentrée, une entrée qui chaque jour est l'occasion d'hésiter sur le pas de la porte... Cesser d'entrer revêt un charme fou. Entrer en classe, c'est entrer dans un système, dans une société, un ordre, faire le deuil de tout ce qui est attirant à l'extérieur. »

23. «L'ambiance est : ici on travaille, le professeur sait que la communauté scolaire va le soutenir dans ce sens-là. Les gens qui auraient plus de difficultés chez nous, ce serait des gens qui ne travailleraient pas, parce qu'en effet, ça ferait tache tout de suite, et leur vie difficile. Ils sont un peu condamnés à travailler » (proviseur).

24. Non seulement, ce terme est de plus en plus spontanément utilisé par les enseignants et les membres de l'équipe d'éducation pour qualifier la conduite professionnelle de leur chef d'établissement, mais on commence à y avoir recours dans l'analyse que l'institution fait d'ellemême : "Tout ce qui précède peut se résumer à un constat simple : un chef d'établissement est le vrai patron. S'il a quelque chose à dire, sa véritable autorité se mesure à la qualité de sa communication. C'est par là qu'il donne vie au projet, par ce qu'il en dit. Sinon il reste un document mort ». Inspection générale de l'Éducation nationale, groupe «Établissements et vie scolaire », Les conseillers principaux d'éducation. Enquête d'Image, Paris, oct. 1992.

25. Dans le panel des chefs d'établissement suivi par le CREDOC, on évalue à $40 \%$ la proportion de ceux qu'on peut qualifier de "managers pédagogiques ", L'opinion des chefs d'établissement sur leur autonomie, Note d'information 93-30, ministère de l'éducation nationale, DEP, juillet 1993. De même, dans le rapport de l'Inspection générale de 1991, on distingue trois profils de proviseur :

- «une première catégorie est composée de proviseurs qui jouent le rôle de locomotive et provoquent un effet d'entraînement (35 \%);

- une seconde catégorie, heureusement peu nombreuse (10\%), aggrave l'inertie ;

- enfin, une troisième catégorie, la plus nombreuse (55\%), fait bien son travail, tant au plan de l'animation que de l'action administrative ».

26. Soulignons que cet interventionnisme est à géométrie variable. Lorsque l'établissement a été « mis en ordre » selon une formulation proposée par J.-L. Derouet, (École et justice, Paris, Métailié, 1992), quand un «fil conducteur » oriente les pratiques individuelles, lorsque par les délégations de compétences se met en place un pilotage collégial, ces chefs d'établissement prennent du 
recul et comparent alors leur rôle, comme nous le dit l'un d'entre eux, à celui d'un "chef d'orchestre qui s'efforce de faire jouer tout le monde en mesure, d'éviter les fausses notes ».

27. G. Mamou, Le chef d'établissement au quotidien : des rôles multiples, CNED de Rouen, CNFPID, 1992.

28. M. Crozier, E. Friedberg, L'Acteur et le Système, Le Seuil, Paris, 1977.

29. M. Mintzberg, Structure et dynamique des organisations (trad.), Ed. d'Organisation, Paris, 1982

\section{RÉSUMÉS}

Le style de direction joue un rôle prépondérant parmi les facteurs d'efficacité d'un établissement scolaire : l'auteur présente ici les conclusions de l'enquête qu'il a menée auprès de vingt-cinq lycées français et, notamment, dégage les principales compétences mises en œuvre par ces « patrons » d'excellence.

INDEX

Index géographique : France

Mots-clés : chef d'établissement, établissement d'enseignement, personnel d'encadrement

\section{AUTEUR}

\section{ROBERT BALLION}

Directeur de recherches, CNRS, France 